\title{
An Evaluation of Oxidative Status in Serum and Breast Milk of Mothers Giving Birth Prematurely and at Full-Term
}

\author{
Mahmut Abuhandan ${ }^{1, *}$; Nihat Demir ${ }^{2}$; Bulent Guzel ${ }^{1}$; Veysi Almaz ${ }^{1}$; Bulent Koca ${ }^{1}$; Oguz \\ Tuncer $^{2}$; Alpay Cakmak ${ }^{1}$ \\ ${ }^{1}$ Department of Pediatrics, Medical Faculty, Harran University, Urfa, Turkey \\ 2 Department of Neonatal Care Unit, Medical Faculty, Yuzuncu Yil University, Van, Turkey \\ *Corresponding author: Mahmut Abuhandan, Department of Pediatrics, Medical Faculty, Harran University, Urfa, Turkey. E-mail: mabuhandan@gmail.com
}

Received: August 22, 2014; Accepted: May 8, 2015

\begin{abstract}
Background: Burning fat and carbohydrates to provide energy in biological systems causes the formation of free oxygen species. Objectives: This study aimed to evaluate the oxidative status of serum and breast milk of mothers giving birth prematurely and at fullterm.

Materials and Methods: The study comprised 50 mothers who gave birth at full-term at more than 38 weeks and 43 mothers who gave birth pre-term at below 32 weeks. On the postnatal 5th day, samples of the mother's milk and serum were taken and stored at $-80^{\circ} \mathrm{C}$ until the study day. On the study day, the total oxidant and total antioxidant levels were measured using the Erel method and the oxidative stress index(OSI) was calculated.

Results: While the total oxidant level and total antioxidant level values of the milk of the premature birth mothers were found to be significantly high compared to those of the full-term birth mothers $(\mathrm{P}=0.001)$, no statistically significant difference was found in the oxidative stress index values $(\mathrm{P}>0.05)$. No statistically significant difference was found in the total oxidant level and oxidative stress index values of the serum of the premature birth mothers compared to those of the full-term birth mothers, while the total antioxidant level was found to be significantly low $(\mathrm{P}=0.04)$.

Conclusions: The oxidants and antioxidants in the milk of mothers giving birth prematurely were found to be significantly higher than those of full-term birth mothers. This can be evaluated as the milk of the premature birth mothers providing increased antioxidant defense to protect the infant.
\end{abstract}

Keywords: Antioxidants; Mother; Breast milk

\section{Background}

Burning fat and carbohydrates to provide energy in biological systems causes the formation of free oxygen species (1). The rate of production of free oxygen species is in balance with the rate of its removal and this status is known as oxidative balance (2). An increase in the rate of production of these radicals or a fall in the rate of removal causes an imbalance (2). If there is an imbalance between the production of free oxygen species and the antioxidant systems, then the structure and functions of proteins, lipids, nucleic acids and enzymes, as elements of the body's fundamental structure, are impaired $(1,3)$, which eventually leads to tissue damage (2). By synthesizing protective enzymes (glutathione reductase, glutathione peroxidase, superoxide dismutase, and catalase) and chemical compounds towards cell protection against the destructive effects of free oxygen species, the formation of damage is prevented $(1,4,5)$. Newborn infants and especially premature infants exposed to high concentrations of oxygen are more vulnerable to infection and inflammation and are thus inclined to the formation of oxidative stress (6, $7)$. Newborns and particularly premature newborns have been shown to have insufficient anti-oxidative capacity against oxygen species in their first days $(8,9)$. The manner of nutrition can affect the anti-oxidant capacity (10). In the postpartum period, the anti-oxidants taken in with the mother's milk try to compensate to neutralize the increased free oxygen species in the infant (11).

\section{Objectives}

In this study, it was aimed to evaluate the serum and milk oxidant and antioxidant status in premature birth mothers and full-term birth mothers.

\section{Materials and Methods}

The study comprised 43 mothers giving birth prematurely at 30 - 32 weeks with no illness and 50 mothers giving birth at full-term at 38 - 42 weeks either by Caesarean section or by normal vaginal route, who had been monitored throughout their pregnancies at the gynecology and obstetrics polyclinics of two health centers between 1 April 2012 and 1 April 2013. The two groups were similar

Copyright (C) 2015, Growth \& Development Research Center. This is an open-access article distributed under the terms of the Creative Commons Attribution-NonCommercial 4.0 International License (http://creativecommons.org/licenses/by-nc/4.0/) which permits copy and redistribute the material just in noncommercial usages, provided the original work is properly cited. 
in terms of age and socio-cultural status. All the mothers had taken supplementary iron and vitamins throughout the pregnancy. On postnatal day 5, the newborn healthy children were called for check-up to the polyclinic, milk, and blood samples were taken from the mothers. Informed consent was obtained from all the mothers participating in the study. Approval for the study was granted by the local ethics committee.

\subsection{Exclusion Criteria}

Mothers with diabetes mellitus, hypertension, obesity, or those who smoked and infants with congenital anomalies, asphyxia in the prenatal or neonatal period or an Apgar score below 8, were excluded from the study. In addition, 5 mothers who did not take supplementary iron and vitamins during pregnancy were excluded.

\subsection{Blood Samples}

Peripheral venous blood samples in the fifth day after birth were taken from all mothers. At the start of the study, total blood count was made by automatic blood count instrument (Abbot Celldyn 3500 Ill, USA) for all the mothers. For biochemical analysis, the blood samples were centrifuged at $3500 \mathrm{rpm}$ for 10 minutes, and then the formed elements were discarded. Part of the serum sample was stored at $-80^{\circ} \mathrm{C}$. The remaining serum was tested for electrolytes, kidney and liver function (Abbott Aeroset, Abbott Diagnostics, Abbott Park, IL, USA) on the same day and on the study day. The total antioxidant status (TAS) and total oxidant status (TOS) were measured color metrically using Erel method by auto-analyzer from the serum samples stored at $-80^{\circ} \mathrm{C}$. The oxidative stress index(OSI) was defined as percentage rate of TAS values to TOS values

\subsection{Homogenization of Milk Samples}

Prior to analysis, all the milk samples were mechanically stirred and homogenized at low speed. The homogenate was centrifuged at $4000 \mathrm{rpm}$ for 10 minutes. The total oxidative status (TOS), TAS, OSI, and microprotein level were measured. The results were given as gram protein.

\subsection{Measurement of Microprotein Level}

The microprotein levels of the milk samples were measured using the Lowry method (12). The microprotein levels of the milk samples were calculated per gram protein for TAS, TOS, and OSI.

\subsection{Measurement of the Total Antioxidant Status}

TAS of plasma was determined using a novel automated measurement method, developed by Erel (13). In this method, the most potent biological radical, hydroxyl radical, is produced. In the assay, ferrous ion solution, which is present in reagent 1 [o-dianisidine $(10 \mathrm{mM})$, ferrous ion (45 AM) in the Clark and Lubs solution ( $75 \mathrm{mM}$, $\mathrm{pH}$ 1.8] is mixed with hydrogen peroxide, which is pres- ent in reagent $2\left[\mathrm{H}_{2} \mathrm{O}_{2}(7.5 \mathrm{mM})\right.$ in the Clark and Lubs solution]. The sequentially produced radicals such as brown colored dianisidinyl radical cation, produced by the hydroxyl radical, are also potent radicals. Using this method, the antioxidative effect of the sample against the potent free radical reactions that is initiated by the produced hydroxyl radical, is measured. The assay has excellent precision values of lower than $3 \%$. The results were expressed as mmol Trolox Equiv. L-1.

\subsection{Measurement of Total Oxidant Status}

TOS of plasma was determined using a novel automated measurement method developed by Erel (14). Oxidants present in the sample oxidize the ferrous ion-o-dianisidine complex to ferric ion. The oxidation reaction is enhanced by glycerol molecules, which are abundantly present in the reaction medium. The ferric ion makes a colored complex with xylenol orange in an acidic medium. The color intensity, which can be measured spectrophotometrically, is related to the total amount of oxidant molecules present in the sample. The assay is calibrated with hydrogen peroxide and the results were expressed in terms of micromolar hydrogen peroxide equivalent per liter ( $1 \mathrm{~mol} \mathrm{H}_{2} \mathrm{O}_{2}$ Equiv. L-1).

\subsection{Oxidative Stress Index}

The ratio percentage of TOS level to TAS level gave the OSI, an indicator of the degree of oxidative stress (15).

\subsection{Statistical Analysis}

Data were analyzed using SPSS (Statistical Package for the Social Sciences, version 11.5 for Windows, SPSS® Inc, Chicago, IL). Distribution of parametric variables was assessed with one-sample Kolmogorov-Smirnov test and all parametric variables were found to be normally distributed. The results were presented as mean \pm standard deviation. Demographic data was performed using chisquare test. Independent Samples t Test was used to data among groups. A two-tailed P value of less than 0.05 was considered statistically significant.

\section{Results}

The mean age of the 43 premature birth mothers was 27.74 \pm 5.70 years (range, 18 - $45 \mathrm{yrs}$ ) and the mean age of the 50 full-term birth mothers was $28.70 \pm 5.76$ years (range, 19 - 40 yrs). No statistically significant difference was determined between the groups in terms of age $(\mathrm{P}>0.05)$ (Table 1$)$.

The TOS, TAS, and OSI values of the milk of the premature birth mothers and the full-term birth mothers were compared. While the TOS and TAS values of the milk of the premature birth mothers were found to be statistically significantly high compared to the TOS and TAS values of the milk of the full-term mothers $(P=0.001)$, no statistically significant difference was found in respect of OSI values $(\mathrm{P}>0.05)$ (Table 1$)$. 
Abuhandan Met al.

Table 1. Demographic Data and TOS, TAS and OSI Values of the Milk of Premature Birth Mothers and Full-Term Birth Mothers a, b,c

\begin{tabular}{lccc}
\hline & Premature Birth Mothers $(\mathbf{n}=\mathbf{4 3})$ & Full Term Birth Mothers $(\mathbf{n}=\mathbf{5 0})$ & PValue $^{\mathrm{d}}$ \\
\hline Age, $\mathbf{y}$ & $27.74 \pm 5.70$ & $28.70 \pm 5.76$ & 0.42 \\
TOS, $\mu \mathbf{m o l ~ H}_{\mathbf{2}} \mathbf{O}_{\mathbf{2}}$ Eqv./gr & $5.42 \pm 1.31$ & $4.56 \pm 1.17$ & 0.001 \\
TAS, $\mu \mathbf{m o l ~ H}_{\mathbf{2}} \mathbf{O}_{\mathbf{2}}$ Eqv./gr & $0.21 \pm 0.03$ & $0.18 \pm 0.02$ & 0.001 \\
OSI, $\mathbf{A U}$ & $2.62 \pm 0.88$ & $2.47 \pm 0.71$ & 0.38 \\
\hline
\end{tabular}

a Abbreviations: OSI, oxidative stress index; TAS, total antioxidant status; TOS, total oxidant status.

$\mathrm{b}$ The data were expressed as mean $\pm \mathrm{SD}$.

c Student $t$ test for independent samples was used

d Significance was defined as $\mathrm{P}<0.05$.

Table 2. Serum TOS, TAS, and OSI Values of Premature Birth Mothers and Full-Term Birth Mothers a,b,c

\begin{tabular}{|c|c|c|c|}
\hline & Premature Birth Mothers $(n=43)$ & Full Term Birth Mothers $(\mathbf{n}=\mathbf{5 0})$ & PValue $^{\mathrm{d}}$ \\
\hline $\mathrm{TOS}, \mu \mathrm{mol} \mathrm{H}_{2} \mathbf{O}_{2}$ Eqv./L & $25.82 \pm 17.07$ & $29.03 \pm 13.54$ & 0.32 \\
\hline TAS, $\mu \mathrm{mol} \mathrm{H}_{2} \mathrm{O}_{2}$ Eqv./L & $1.29 \pm 0.15$ & $1.36 \pm 0.16$ & 0.04 \\
\hline OSI, AU & $1.97 \pm 1.21$ & $2.16 \pm 1.03$ & 0.40 \\
\hline
\end{tabular}

a Abbreviations: OSI, oxidative stress index; TAS, total antioxidant status; TOS, total oxidant status.

$\mathrm{b}$ The data were expressed as mean $\pm \mathrm{SD}$.

c Student $t$ test for independent samples was used.

$\mathrm{d}$ Significance was defined as $\mathrm{P}<0.05$.

The serum TOS, TAS, and OSI values of the premature birth mothers and the full-term birth mothers were compared. While the serum TAS values of the premature birth mothers were found to be statistically significantly low compared to the serum TAS values of the full-term mothers $(P=0.04)$, no statistically significant difference was found in respect of TOS and OSI values $(\mathrm{P}>0.05)$ (Table 2).

\section{Discussion}

The metabolism employs its own anti-oxidant defense systems against oxidative effects. The removal of reactive oxygen species and rendering them harmless is accomplished through enzymatic and non-enzymatic anti-oxidant mechanisms (15). Many studies to date have shown that nutrition with mother's milk is a protection against many diseases. One of these protective mechanisms is undoubtedly anti-oxidant defense (16-20). Newborn infants and particularly premature newborns have been shown to have insufficient anti-oxidative capacity against free oxygen species in their first days $(8,9)$.

It has been reported that the number and increased activity of anti-oxidant enzymes occur in the final months of pregnancy and after the birth, the total antioxidant capacity increases rapidly to reach levels higher than that of adults $(9,21)$. Studies are ongoing on the subject of the effect of breastfeeding on the oxidative-anti-oxidative system of infants and the development of diseases $(10,19$, 22). In the current study, while the TOS and TAS values of premature birth mothers were found to be statistically significantly high compared to those of full-term birth mothers, no statistically significant difference was found in respect of OSI values.
As the pregnancy progresses, together with adaptive changes in the functions of the mother's body to meet the requirements of the fetus $(23,24)$; ventilation increases to meet the increasing demand for oxygen (25, 26). This could be a reason for increased oxidative stress in pregnancy (27). In addition, the higher levels of psychosocial stress experienced by premature birth mothers $(28,29)$, such as feelings of failure and insecurity at not having given birth to a healthy infant (28) and difficulties experienced in bonding, such as being slow to touch and nurse the baby or not perceiving the baby as their own $(28,29)$ may cause increased oxidative stress associated with stress in the mother.

The increase in anti-oxidants may be necessary to neutralize the increased oxidative status of the mother. As newborn infants and particularly premature newborns do not have insufficient anti-oxidative capacity against free oxygen species in their first days $(8,9)$, it is thought that anti-oxidants in the milk of premature birth mothers increase to protect the infant.

In a study evaluating the serum oxidant and anti-oxidant status of pregnant and non-pregnant women, the oxidative stress of the pregnant women was found to be significantly high and the antioxidants were significantly low (27). In the same study, it was reported that apart from ascorbic acid, both oxidants, and anti-oxidants in the mothers were at a significantly high level compared to those of the infants (27). In the current study, the serum TOS, TAS, and OSI values were compared in premature birth mothers and full-term birth mothers.

While no statistically significant difference was found in the serum TOS and OSI values of the premature birth 
mothers compared to those of full-term mothers, the TAS values were found to be statistically significantly low. It is thought that because in this situation where newborn infants are exposed to a high concentration of oxygen they have a tendency to form oxidative stress $(6,7)$ and newborns' anti-oxidative capacity is insufficient against oxygen species in the first days so antioxidants are produced by the mother to protect the infant. In addition, it is thought that the oxidant production of the full-term birth mothers is at a low level as they experience less anxiety about the newborn.

In conclusion, many studies have shown that mother's milk is protective against several diseases. One of these protective mechanisms is undoubtedly the anti-oxidant defense. Newborn infants and especially premature infants exposed to high concentrations of oxygen are more vulnerable to infection and inflammation and are thus inclined to the formation of oxidative stress, so it is thought that the mother's milk, especially that of premature birth mothers, raises the antioxidant defense to protect the infant whose antioxidant capacity is insufficient.

\section{References}

1. Scandalios JG. The rise of ROS. Trends Biochem Sci. 2002;27(9):483-6.

2. Serafini M, Del Rio D. Understanding the association between dietary antioxidants, redox status and disease: is the Total Antioxidant Capacity the right tool? Redox Rep. 2004;9(3):145-52.

3. Yigit S, Yurdakok M, Kilin K, Oran O, Erdem G, Tekinalp G. Serum malondialdehyde concentration in babies with hyperbilirubinaemia. Arch Dis Child Fetal Neonatal Ed.1999;80(3):F235-7.

4. Janero DR. Malondialdehyde and thiobarbituric acid-reactivity as diagnostic indices of lipid peroxidation and peroxidative tissue injury. Free Radic Biol Med.1990;9(6):515-40.

5. Halliwell B. Antioxidant characterization. Biochem Pharmacol. 1995;49(10):1341-8.

6. Gitto E, Reiter RJ, Karbownik M, Tan DX, Gitto P, Barberi S, et al. Causes of oxidative stress in the pre- and perinatal period. Biol Neonate. 2002;81(3):146-57.

7. Saugstad OD. Bronchopulmonary dysplasia-oxidative stress and antioxidants. Semin Neonatol. 2003;8(1):39-49.

8. Georgeson GD, Szony BJ, Streitman K, Varga IS, Kovacs A, Kovacs $\mathrm{L}$, et al. Antioxidant enzyme activities are decreased in preterm infants and in neonates born via caesarean section. Eur J Obstet Gynecol Reprod Biol. 2002;103(2):136-9.

9. Robles R, Palomino N, Robles A. Oxidative stress in the neonate. Early Hum Dev. 2001;65 Suppl:S75-81.

10. Aycicek A, Erel O, Kocyigit A, Selek S, Demirkol MR. Breast milk provides better antioxidant power than does formula. Nutrition. 2006;22(6):616-9.

11. Friel JK, Martin SM, Langdon M, Herzberg GR, Buettner GR. Milk from mothers of both premature and full-term infants provides better antioxidant protection than does infant formula. Pediatr Res. 2002;51(5):612-8.

12. Lowry OH, Rosebrough NJ, Farr AL, Randall RJ. Protein measurement with the Folin phenol reagent. JBiol Chem.1951;193(1):26575.

13. Erel O. A novel automated method to measure total antioxidant response against potent free radical reactions. Clin Biochem. 2004;37(2):112-9.

14. Erel O. A new automated colorimetric method for measuring total oxidant status. Clin Biochem. 2005;38(12):1103-11.

15. Halliwell B. Free radicals, antioxidants, and human disease: curiosity, cause, or consequence? Lancet.1994;344(8924):721-4.

16. Ahola T, Fellman V, Kjellmer I, Raivio KO, Lapatto R. Plasma 8-isoprostane is increased in preterm infants who develop bronchopulmonary dysplasia or periventricular leukomalacia. Pediatr Res. 2004;56(1):88-93.

17. Back SA, Luo NL, Mallinson RA, O'Malley JP, Wallen LD, Frei B, et al. Selective vulnerability of preterm white matter to oxidative damage defined by F2-isoprostanes. Ann Neurol. 2005;58(1):108-20.

18. Kermorvant-Duchemin E, Sennlaub F, Chemtob S, Behar-Cohen F. [Pathophysiology of retinopathy of prematurity]. Arch Pediatr. 2006;13(6):566-8.

19. Ezaki S, Ito T, Suzuki K, Tamura M. Association between Total An tioxidant Capacity in Breast Milk and Postnatal Age in Days in Premature Infants. JClin Biochem Nutr. 2008;42(2):133-7.

20. Hanna N, Ahmed K, Anwar M, Petrova A, Hiatt M, Hegyi T. Effect of storage on breast milk antioxidant activity. Arch Dis Child Fetal Neonatal Ed. 2004;89(6):F518-20.

21. Wiedemann M, Kontush A, Finckh B, Hellwege HH, Kohlschutter A. Neonatal blood plasma is less susceptible to oxidation than adult plasma owing to its higher content of bilirubin and lower content of oxidizable Fatty acids. Pediatr Res. 2003;53(5):843-9.

22. Sommerburg O, Meissner K, Nelle M, Lenhartz H, Leichsenring M Carotenoid supply in breast-fed and formula-fed neonates. Eur Pediatr. 2000;159(1-2):86-90.

23. Weissgerber TL, Wolfe LA. Physiological adaptation in early human pregnancy: adaptation to balance maternal-fetal demands. Appl Physiol Nutr Metab. 2006;31(1):1-11.

24. Clapp J3, Seaward BL, Sleamaker RH, Hiser J. Maternal physiologic adaptations to early human pregnancy. Am J Obstet Gynecol. 1988;159(6):1456-60.

25. Alaily AB, Carrol KB. Pulmonary ventilation in pregnancy. BrJ Obstet Gynaecol.1978;85(7):518-24.

26. Pernoll ML, Metcalfe J, Kovach PA, Wachtel R, Dunham MJ. Ventilation during rest and exercise in pregnancy and postpartum. Respir Physiol.1975;25(3):295-310.

27. Upadhyaya C, Mishra S, Singh PP, Sharma P. Antioxidant status and peroxidative stress in mother and newborn -A pilot study. Indian JClin Biochem. 2005;20(1):30-4.

28. Young Seideman R, Watson MA, Corff KE, Odle P, Haase J, Bowerman JL. Parent stress and coping in NICU and PICU.J Pediatr Nurs. 1997;12(3):169-77.

29. Gibbins SA, Chapman JS. Holding on: parents' perceptions of premature infants' transfers. J Obstet Gynecol Neonatal Nurs. 1996;25(2):147-53. 